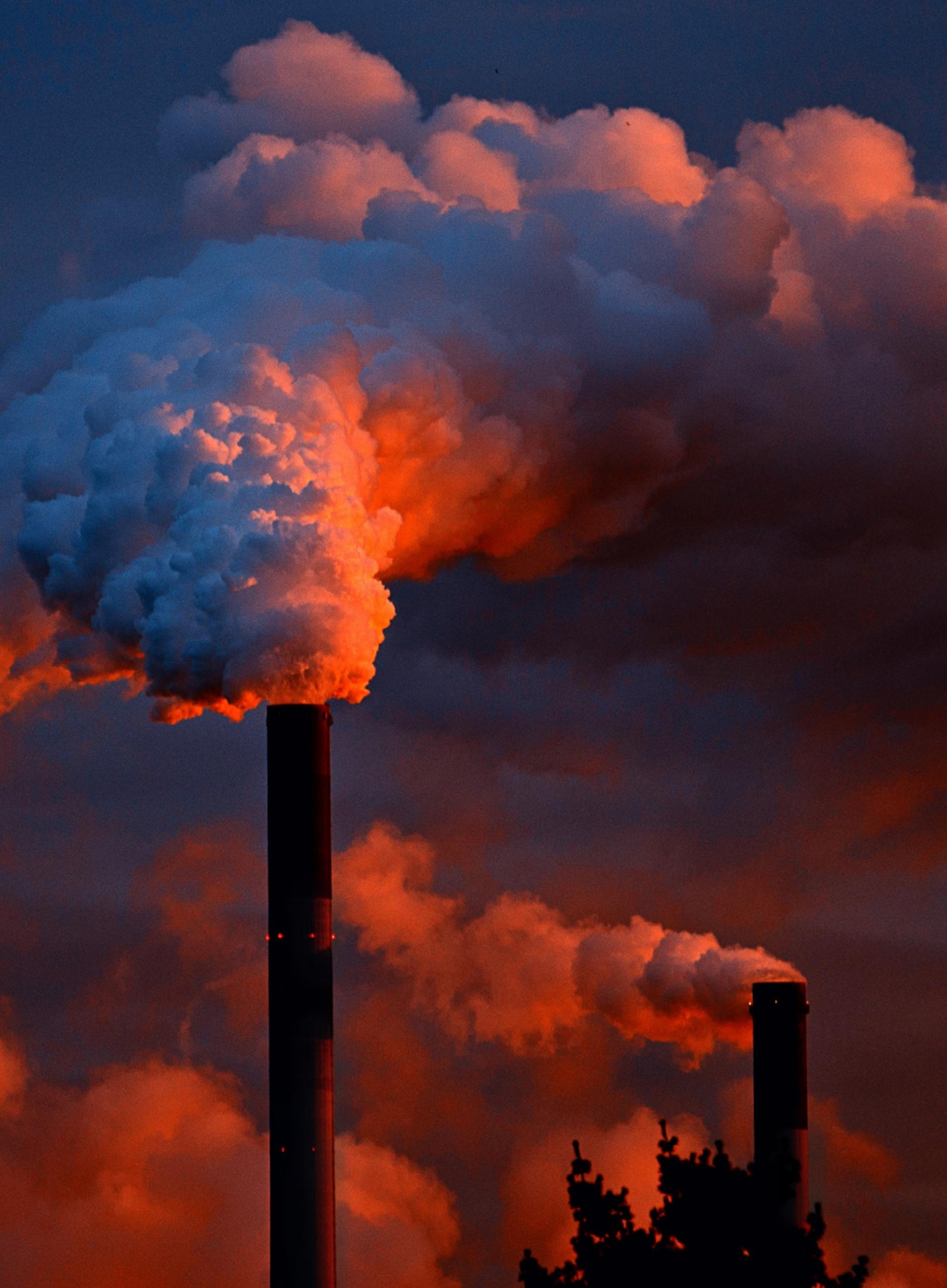



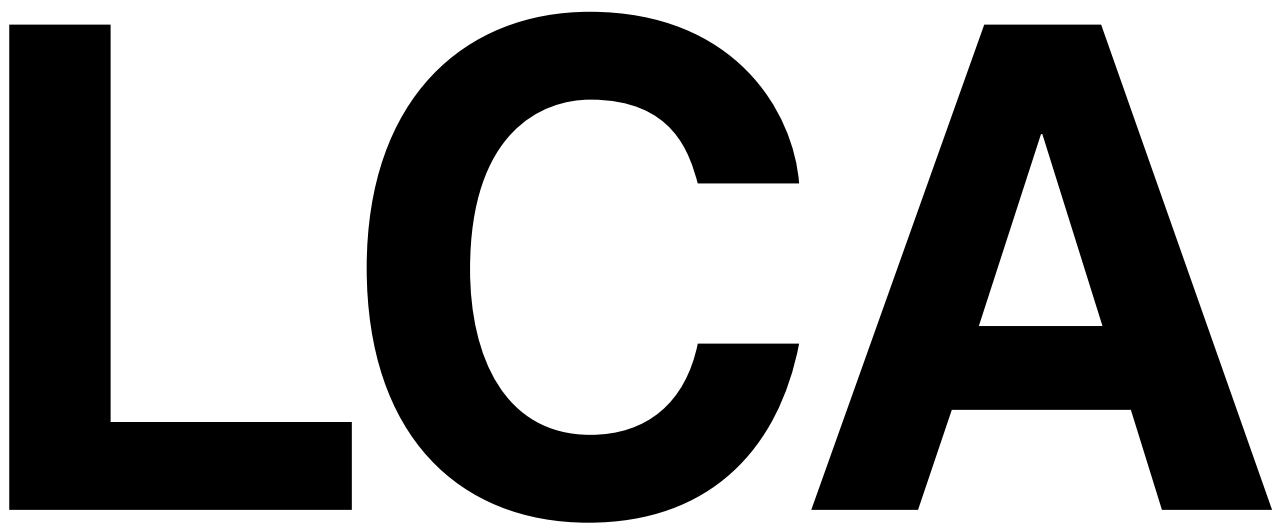

\section{El Laboratorio de Contaminación Atmosférica de la UMH}

BELÉN PARDOS

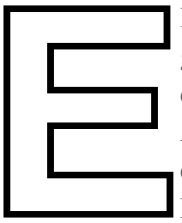

grupo de investigación Laboratorio de Contaminación Atmosférica (LCA) de la Universidad Miguel Hernández

(UMH) de Elche tiene como objetivo principal de su labor científica la caracterización del aire ambiente en la vertiente mediterránea española $y$, en concreto, en la provincia de Alicante. Desde hace alrededor de siete años, el LCA mantiene en funcionamiento una estación de medida de la calidad del aire, ubicada en la cima de la sierra de Aitana, el pico más alto de la provincia de Alicante. De los datos obtenidos, deriva un conocimiento más detallado de la naturaleza, origen, variabilidad y dinámica de los principales contaminantes atmosféricos para mejorar la comprensión de sus efectos sobre la salud, el medio ambiente y el clima en el Mediterráneo occidental.

La ubicación de la estación de medida en la cima de Aitana tiene su porqué en la necesidad de obtener datos sobre los niveles ambientales de fondo remoto regional de los contaminantes atmosféricos en la provincia de Ali- cante. El responsable del grupo, Javier Crespo, explica que se trata de un lugar de interés científico por su singularidad, a una altura de casi 1.600 metros y a solo unos 15 kilómetros del Mar Mediterráneo: "Las otras cimas se encuentran muy al interior respecto de la costa, por eso éste es un lugar idóneo para estudiar las características del aire de fondo limpio muy cercano al Mediterráneo". Aunque puntualiza que, en la actualidad, el aire realmente limpio no existe. La importancia de la referencia de Aitana es que permite comparar el aire más limpio posible con el de diferentes ambientes urbanos del entorno, donde la calidad del aire se ha modificado en mayor

El grupo
analizala
calidad del aire
condudes yemi-
zonanas de la
provincia

ambientales de eventos naturales frecuentes en la zona, como las intrusiones de masas de aire procedentes del norte de África que alteran los niveles y las propiedades del aerosol y otros contaminantes.

Así, los datos obtenidos en la estación de Aitana se comparan regularmente con otros tomados en ciudades como Elche o Alicante. De esta manera, el LCA puede analizar o menor medida como consecuencia de la actividad humana. Otra razón que subraya la utilidad de la estación de Aitana es que permite detectar y estudiar, sin la interferencia de emisiones antropogénicas, los impactos cómo se encuentra y evoluciona la calidad del aire en ciudades y zonas semiurbanas de la provincia. Entre la tecnología empleada se utilizan muestreadores manuales que recogen las partículas en suspensión atmosférica -aerosol-sobre filtros de cuarzo o teflón, para analizar su composición química. Por su parte, los equipos automáticos facilitan datos de concentración de contaminantes en tiempo real, como el ozono o el dióxido de nitrógeno. "De manera que podemos conocer, por ejemplo, la cantidad de partículas que se encuentran en el aire, según su tamaño, en un periodo concreto", asegura Crespo. Además, el LCA dispone de diferentes aparatos para medir parámetros ópticos característicos del aerosol, como los coeficientes de absorción o de difusión, que sirven para estudiar su contribución al calentamiento de la atmósfera.

La estación de Aitana dispone, también, de una torre meteorológica con la que se registran parámetros como la dirección y la velocidad del viento, la radiación solar total, la temperatura, la humedad relativa o la pluviometría. "Los equipos funcionan continuamente $\mathrm{y}$, a través de una antena de comunicaciones, vía radio enlace, tenemos conexión por internet con la Universidad. En la mayoría de los casos, es posible la observación y la descarga de datos en tiempo real", subraya Javier Crespo. El profesor de la UMH incide en la importancia de poder mantener activa una estación como la de Aitana durante mucho tiempo: "Necesitamos largas series de datos, continuas en el tiempo, para poder determinar > 


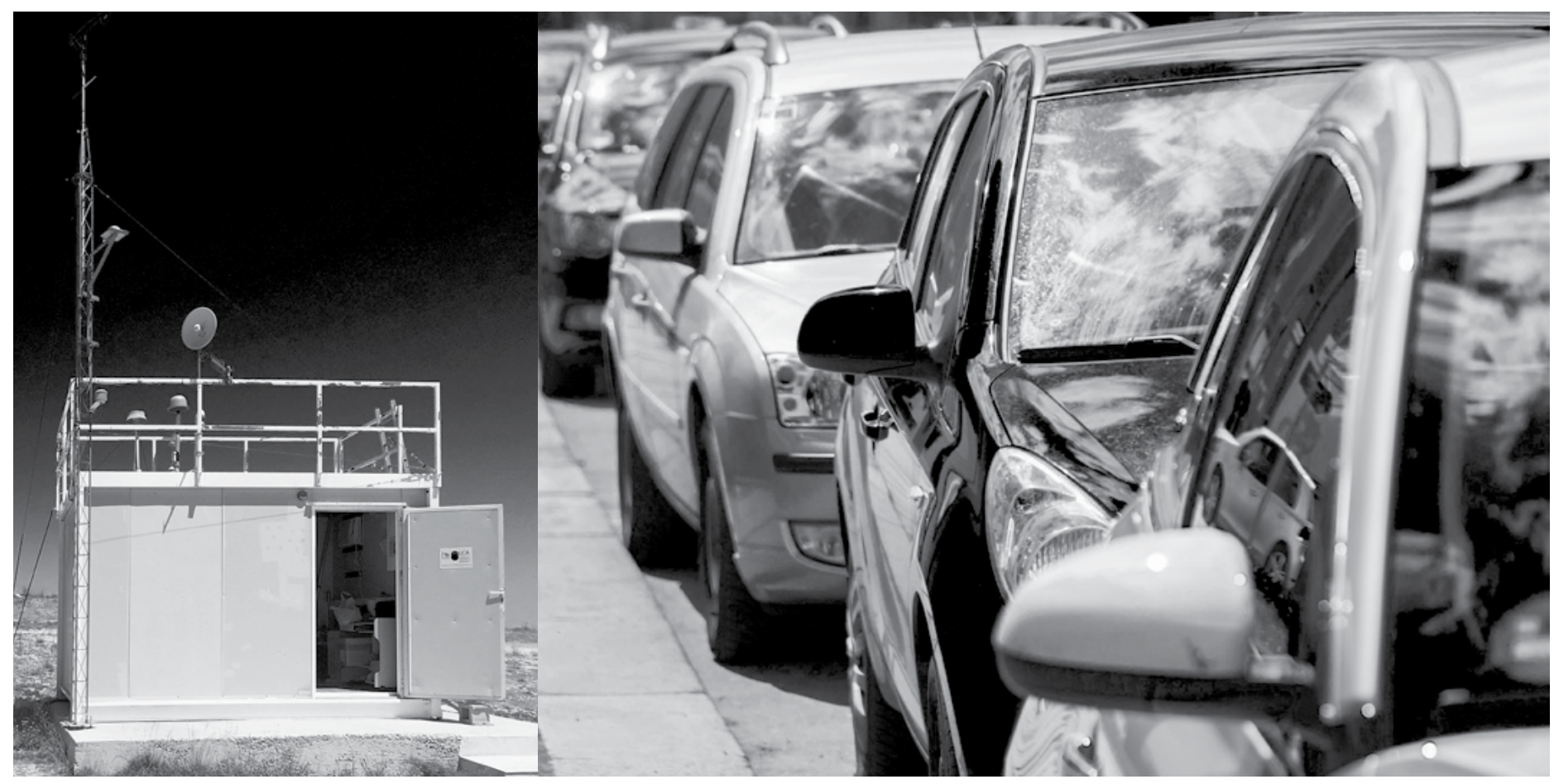

> tendencias desde el punto de vista de la calidad del aire. Por ejemplo, para estudiar de forma precisa el impacto de los aerosoles atmosféricos en el cambio climático".

\section{El aire en Alicante}

El responsable del LCA señala que la provincia de Alicante, por sus características geográficas, meteorológicas y humanas, tiene dos contaminantes atmosféricos principales: los aerosoles -partículas en suspensión en el aire- y el ozono troposférico. En el primer caso, debido a la baja pluviometría y a las características de los suelos de la zona casi desprovistos de vegetación y pedregosos, existe una elevada carga de material particulado en la atmósfera. El ozono troposférico, sustancia muy tóxica, se origina en la parte baja de la atmósfera, por el efecto combinado de una intensa irradiación solar, alta densidad de tráfico y elevada temperatura, factores habituales, sobre todo, en el verano alicantino.

Aunque estos dos contaminantes son los de niveles relativos mayores en la provincia, en las ciudades se puede encontrar, de forma puntual, niveles notables de óxidos de nitrógeno que

\section{Javier Crespo subraya la importancia de obtener datos durante largos periodos de tiempo}

proceden fundamentalmente de los vehículos con motores de combustión, originados por la actividad humana En el centro de Elche, por ejemplo, el grupo dispone de equipos instalados con el objetivo de medir los niveles de concentración de contaminantes originados por el tráfico.

Javier Crespo apunta que, en términos generales, la calidad del aire en Alicante es aceptable ya que no existen grandes industrias pesadas $\mathrm{y}$, además, los focos principales de contaminación, las ciudades de mayor población, se encuentran próximos a la costa mediterránea: "El régimen típico de brisas, dispersa los contaminantes, aunque existen zonas en las ciudades un poco más conflictivas".

Una de las últimas colaboraciones científicas llevadas a cabo por el LCA está relacionada con un estudio que se desarrolla en el puerto de Alicante sobre la manipulación, carga y descarga de graneles industriales. El grupo analizará cómo influye la actuación y las actividades del puerto en los niveles de material particulado que afecta al entorno ciudadano limítrofe con el puerto

El director de grupo asegura que su objetivo inmediato es continuar obteniendo financiación para poder mantener los sistemas en funcionamiento. "Si tomamos medidas durante 3 años y después nos vemos obligados a parar por falta de presupuesto, el esfuerzo realizado no sirve de nada", asevera. Por este motivo, Javier Crespo asegura que resulta fundamental la colaboración entre Universidad, empresas e instituciones para mantener la investigación y así contribuir a ampliar los límites del conocimiento •

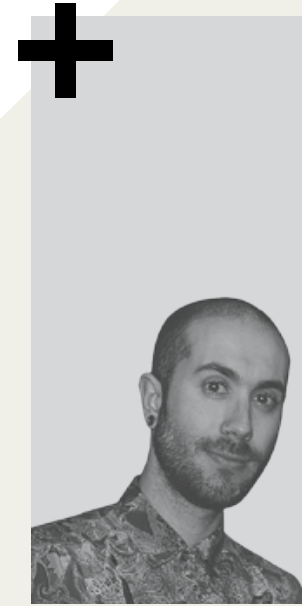

\section{La concentración de contaminantes en alta montaña}

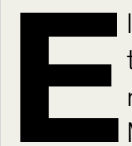

doctor en Análisis y Gestión Ambiental por la Universidad Miguel Hernández (UMH) de Elche Rubén Soler Moreno ha caracterizado en su tesis doctoral el material particulado atmosférico y el ozono en la sierra de Aitana. El objetivo del estudio pasa por analizar el impacto que tienen ciertos episodios asociados a distintos procesos de transporte de masas de aire sobre las concentraciones de contaminantes registradas en la estación de montaña.

\section{El trabajo de Soler Moreno revela que,} cuando coinciden determinados factores meteorológicos, se puede propiciar que episodios de polución urbanos, asociados a situaciones de elevada estabilidad atmosférica durante los meses más fríos, incidan sobre las concentraciones de partículas en puntos de alta montaña. El autor de la tesis explica que, a pesar de que este fenómeno se ve dificultado por la complejidad de la orografía, se ha observado que el transporte de contaminantes es efectivo cuando confluyen una serie de factores: fuerte emisión y acumulación de contaminantes en las zonas urbanas costeras; brisas marinas y de ladera consistentes y favorables, que posibiliten el arrastre y transporte de estos contaminantes hacia la cima; residencia de la cumbre de Aitana dentro de la capa límite de mezcla, donde se da un flujo turbulento de masas de aire y un transporte eficiente a nivel regional.

La tesis concluye que este tipo de transporte conlleva un importante impacto sobre la concentración del material particulado fino. "Al contrario de lo que pudiese pensarse debido a la granulometría predominantemente gruesa de las partículas transportadas durante la intrusión", aclara Soler. Durante estos eventos, además, se ha registrado un incremento de los niveles de carbono orgánico y sulfatos de amonio, debido, por un lado, a una formación secundaria en la atmósfera y, por otro, a una posible incorporación de estos compuestos a las masas de aire a su paso por zonas en las que se han dado episodios previos de formación y acumulación de contaminantes.

El estudio ha servido para verificar que la interacción de las corrientes de aire con el polvo mineral conlleva un impacto sobre los niveles de ozono troposférico, contaminante en la capa inferior de la atmósfera. En concreto, las concentraciones de ozono descendieron en un 5,5\% de media durante todo el periodo de intrusiones de polvo sahariano y llegaron a manifestar reducciones superiores al 15\% en algunas de ellas. 